\title{
Research on the Weight Removing Model and Control Method for the Unbalance Vector of Thin Disc Workpiece
}

\author{
Shihai Zhang $(\mathbb{D}$, Yecheng Zhu $(\mathbb{D}$, and Zimiao Zhang $(\mathbb{D}$ \\ College of Mechanical Engineering, Tianjin University of Technology and Education, Tianjin 300222, China \\ Correspondence should be addressed to Shihai Zhang; zshky77@163.com
}

Received 16 September 2021; Accepted 6 January 2022; Published 28 January 2022

Academic Editor: Jussi Sopanen

Copyright ( $\odot 2022$ Shihai Zhang et al. This is an open access article distributed under the Creative Commons Attribution License, which permits unrestricted use, distribution, and reproduction in any medium, provided the original work is properly cited.

\begin{abstract}
Aiming at the structuring characteristics of thin disc workpiece, we propose the weight removing model and control methods to reduce the mass unbalance vector. Based on the material removing and correcting principle for unbalance workpiece, the weight removing model of drilling and reaming hole way and internal and external arc milling is discussed. The iterative control method with variable weight removing rate is proposed to reduce the weight removing material of the first-time correction and improve the ultimate correcting accuracy. The online measuring and correcting feasibility for unbalance workpiece is verified by experimental way. The advantages of the proposed iterative control method are discussed by simulation analysis. The research results can be used for the online correction of the unbalance disc workpiece on the NC machine tools or automatic dynamic balancing machine.
\end{abstract}

\section{Introduction}

The disc component with large diameter-thickness ratio is the common structural type of rotor system, since the rotating radius of disc component is large, the greater unbalance vector may be formed even if the unbalance mass is small, and the whole balance performances of rotor system will be greatly influenced by the unbalance components. Therefore, the dynamic balancing machine is widely used to measure and correct the unbalance vector before the disc components are assembled. The problems of high balance cost, low balance efficiency, potential installation, and position errors are unavoidable based on the method of the dynamic balancing machine. According to above problems, an online balance method for thin disc workpiece is proposed in our research. The online measuring and correcting principles for the unbalance vector of thin disc workpiece are discussed as follows.

The online measuring principle is that the initial unbalance vector of machine tool spindle is firstly measured. In the workpiece processing stage, the synthetic unbalance vector of spindle and workpiece is measured online after the workpiece has been machined, and then the residual unbalance vector can be estimated by analyzing the unbalance vibration difference of spindle before and after the thin disc workpiece is clamped. The main problems of online measurement for unbalance disc workpiece have been discussed in [1].

After the unbalance vector of thin disc workpiece has been measured, it is unnecessary that the workpiece is removed from machine tool for correction, and the spindle accurate stopping, tool positioning, and cutting functions of compound $\mathrm{CNC}$ machine tool are used to accurately remove the unbalance vector of workpiece. The key problems of online correction for unbalance disc workpiece are discussed in the paper.

After the unbalance vector of workpiece has been measured, the weight removing way is the common method used to correct the unbalance component on the NC machine tools or automatic dynamic balancing machine. Aiming at the laser processing way, M. Stoesslein et al. developed a laser balancing system to continuously remove material from a target part in a controlled and automated manner [2]. M. Stoesslein et al. introduced the concept of on-the-fly laser machining, and the overall concept was validated by balancing a rotor system through ablation of different pocket shapes by the use of a Yb:YAG pulsed fiber 
laser [3]. The machining ways are mainly applied to automatic dynamic balancing machine at present. In order to improve productivity efficiency and the one-cut success rate of the V-shaped milling unbalance correction of the automatic rotor balancing machine, $\mathrm{S}$. Zeng et al. discussed the modeling method of $\mathrm{V}$-shaped milling unbalance correction and the real-time parameter interpolation problems, and an accurate full model was presented and simplified in a very complex integral mode [4]. S.M. Yin et al. studied the model of R-shaped milling unbalance correction [5]. According to the impact of the rotor alveolar on milling mass and milling position, a mathematical model based on Newton iteration method was used to compensate for correct phase error and amplitude error appropriately.

Aiming at solving the control problem of workpiece dynamic balance, Y.A. Khulief et al. presented a scheme that combined both the influence coefficients and modal balancing techniques. The scheme was developed for low-speed balancing of high-speed rotors and relied on knowledge of the modal characteristics of the rotor [6]. J. Zhang et al. proposed an influence coefficient calibration and online updating method based on hierarchical Bayesian method for automatic dynamic balancing machine [7]. H.W. Fan et al. established a mathematical model of influence coefficient table method for rotor online active balance, where the control stability was analyzed and implementation scheme was designed [8]. L. Zhang et al. designed a feedforward gain adaptive controller to improve the balance control of rotor system under both variable speed and constant speed [9]. F.B. Becker et al. designed the linear gain-scheduled $\mathrm{H}$-infinity controllers guaranteeing both robust stability and performance within a given range of operating conditions [10]. J. Xu et al. proposed a fuzzy self-tuning single neuron PID control method to improve the control efficiency and balance accuracy of rotor automatic balance [11]. In the unbalance correction processing of disc component, influenced by the measuring, correcting, installing, and positioning errors, it is difficult to ensure that the residual unbalance vector meet the requirements of balance accuracy by one-time correcting method. Therefore, the iterative correcting method is used to repeatedly reduce the residual unbalance vector. As for the correcting way of weight removing, the removed material can be regenerated, and the workpiece may be destroyed by the repeated correction when the errors are excessive. Consequently, it is significant to research the new iterative control method for the weight removing way.

In the article, the thin disc workpiece is taken as the researching object. Based on the research results of online measuring and estimating methods for the unbalance vector of thin disc workpiece in [1], the key problems of weight removing model and control method are discussed and used to correct the unbalance vector of thin disc workpiece.

\section{The Weight Removing Model for Unbalance Thin Disc Workpiece}

The purpose of the weight removing model is to convert the unbalance vector of workpiece into the cutting volume of the specify location on workpiece, and then the tool feed rate and path can be calculated and designed.

2.1. The Weight Removing Model of Drilling Hole Way. After the unbalance vector of thin disc workpiece has been measured and calculated, the suitable volume material in the selected position of workpiece can be removed by drilling hole way, and then the unbalance vector of workpiece is corrected. The physical models of the drilling hole way are shown in Figure 1.

Usually, the tool radius cannot be set arbitrarily and is only selected from tool library, so the key problem of weight removing model is how to calculate the drilling depth in the cutting position. The weight removing model steps of drilling hole way are shown as follows:

(1) The measured unbalance vector of thin disc workpiece is expressed as the form of polar coordinates.

$$
\begin{aligned}
\vec{U} & =U \angle \theta \\
& =m r \angle \theta .
\end{aligned}
$$

In (1), $\theta$ is the phase of unbalance vector based on the reference point on spindle, and the weight removing direction depends on the phase. $U$ is the amplitude of unbalance vector, and the weight removing mass or volume depends on the amplitude. The distance $(r)$ between the weight removing hole and workpiece center can be determined reasonably, and then the weight removing mass $(m)$ can be calculated.

(2) According to the density $(\rho)$ of the workpiece material, the weight removing volume $(v)$ can be calculated and expressed in the following equation:

$$
v=\frac{m}{\rho} \text {. }
$$

Referring to Figure 1(c), the radius and apex angle of tool are, respectively, assumed to be $R$ and $\gamma$. The weight removing volume $\left(v_{1}\right)$ of the tool conical part can be expressed as follows:

$$
v_{1}=\frac{1}{3} \frac{\pi R^{3}}{\tan 0.5 \gamma} .
$$

The cutting depth $(y)$ can be calculated by the following equation:

$$
\left\{\begin{array}{l}
i f \mid v \leq v_{1}, \quad y=\sqrt[3]{3 v / \tan ^{2} 0.5 \gamma} \\
i f \mid v>v_{1}, \quad y=\frac{R}{\tan 0.5 \gamma}+\frac{v-v_{1}}{\pi R^{2}}
\end{array}\right.
$$

(3) After the cutting depth of tool has been calculated, the rotating spindle with unbalance workpiece is accurately stopped at the reference position firstly, then the tool is controlled to the weight removing position, and the unbalance mass of workpiece can be removed online. 


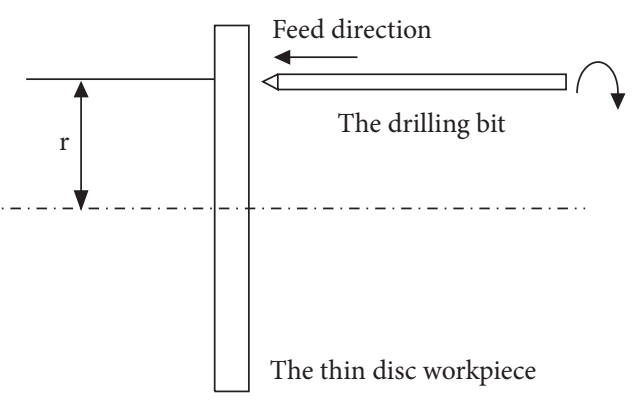

(a)

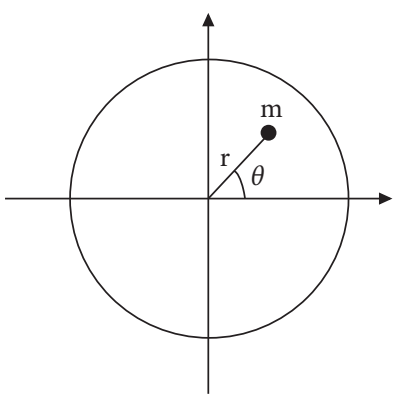

(b)

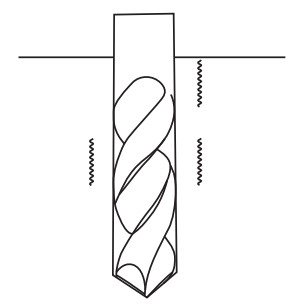

(d)

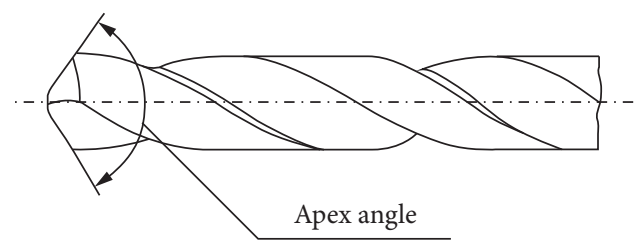

(c)

FIGURE 1: The physical models of drilling hole way: (a) the weight removing principle by drilling hole way; (b) the weight removing position; (c) the tool structure; (d) the cutting process.

2.2. The Weight Removing Model of Reaming Hole Way. For some workpieces, there are several unthreaded holes uniformly distributed in the workpiece, and these holes can be used to remove weight by reaming way. The physical models of the reaming hole way are shown in Figure 2.

In Figure 2, there are six unthreaded holes uniformly distributed in the workpiece, $\vec{U}$ is the equivalent unbalance vector of the workpiece, $R_{0}$ is the initial radius of the hole, and $L$ is the radius of the holes distributed circle. The weight removing model steps of reaming hole way are shown as follows:

(1) According to the phase of workpiece equivalent unbalance vector, one or more holes are selected for reaming. Taking Figure 2 as an example, we select holes 1 and 2 for reaming.

(2) Based on Figure 2, the follows equation relations can be gained according to vector composition principle.

$$
\left\{\begin{array}{l}
\vec{U}=\sum\left(\vec{U}_{1}+\vec{U}_{2}\right), \\
U_{1}=\rho \pi\left(R^{2}-R_{0}^{2}\right) h_{1} L \angle \theta_{1}, \\
U_{2}=\rho \pi\left(R^{2}-R_{0}^{2}\right) h_{2} L \angle \theta_{2} .
\end{array}\right.
$$

In (5), $\vec{U}_{1}$ and $\vec{U}_{2}$ are, respectively, the equivalent unbalance vectors of the expected removing material of holes 1 and $2 ; \rho$ is the density of the workpiece material; $h_{1}$ and $h_{2}$ are, respectively, the reaming depths of holes 1 and 2; $R$ is the tool radius (can be selected from tool library by estimating method). Taking $x$-axis as the reference, we can calculate the phases of $\theta_{1}$ and $\theta_{2}$ according to the positions of holes 1 and 2. The unbalance vector $\vec{U}$ is equivalently decomposed to $x$-axis vector $\left(\vec{U}_{x}\right)$ and $y$-axis vector $\left(\vec{U}_{y}\right)$, and the following equation can be obtained.

$$
\left\{\begin{array}{l}
u_{x}=m r \cos \theta=\rho \pi\left(R^{2}-R_{0}^{2}\right) L\left(h_{1} \cos \theta_{1}+h_{2} \cos \theta_{2}\right), \\
u_{y}=m r \sin \theta=\rho \pi\left(R^{2}-R_{0}^{2}\right) L\left(h_{1} \sin \theta_{1}+h_{2} \sin \theta_{2}\right) .
\end{array}\right.
$$

Based on (6), the reaming depths $h_{1}$ and $h_{2}$ can be calculated, and then the tool can be controlled to remove suitable material according the calculated depths. In some special cases, if the calculated depths are greater than the original depths of holes, the tool with larger diameter or more holes can be selected for reaming.

2.3. The Weight Removing Model of Internal or External Arc Milling Way. The arc milling way can be used to remove the unbalance material for the disc workpiece with internal or external arc edge. Usually, the weight removing arc edge is not the working face. The typical arc edge for weight removing is shown in Figure 3.

In Figure 3, it can be defined, respectively, as the internal and external arc milling if arc faces 1 and 2 are selected correspondingly as the weight removing faces. The geometric models of internal and external arc milling are shown in Figure 4.

In Figure 4(a), $R_{1}$ and $\mathrm{O}_{1}$ are, respectively, assumed to be the radius and the center of the arc milling face; $R_{2}$ and $\mathrm{O}_{2}$ are, respectively, assumed to be the radius and the center of the circular tool path; and $e$ is the eccentric distance between $\mathrm{O}_{1}$ and $\mathrm{O}_{2}$. The intersection points of the arc milling face and the circular tool path are, respectively, named as A and B. $\theta_{1}$ is the included angle between the $\mathrm{O}_{1} \mathrm{~A}$ and $y$-axis, and $\theta_{2}$ is the included angle between the $\mathrm{O}_{2} \mathrm{~A}$ and $y$-axis.

In Figure 4(b), $R_{2}$ and $\mathrm{O}_{2}$ are, respectively, assumed to be the radius and the center of the arc milling face; $R_{1}$ and $\mathrm{O}_{1}$ are, respectively, assumed to be the radius and the center of the circular tool path; and other assumptions are similar to Figure 4(a). 


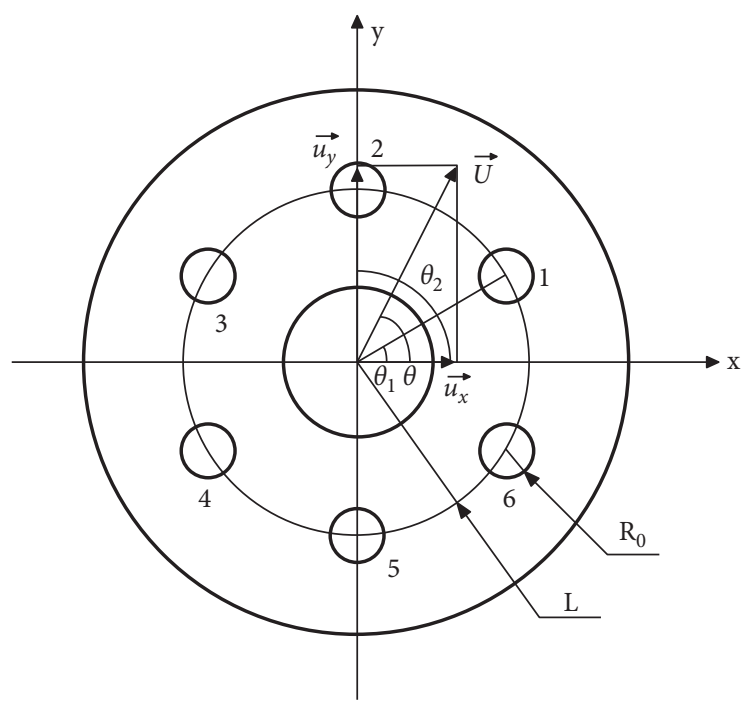

Figure 2: The physical models of reaming hole way.

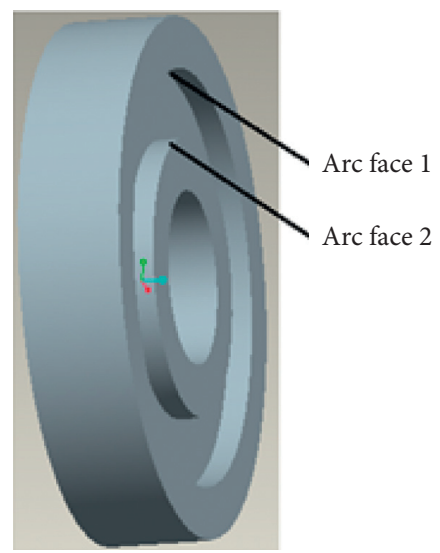

FIgURE 3: The typical arc edge for weight removing.

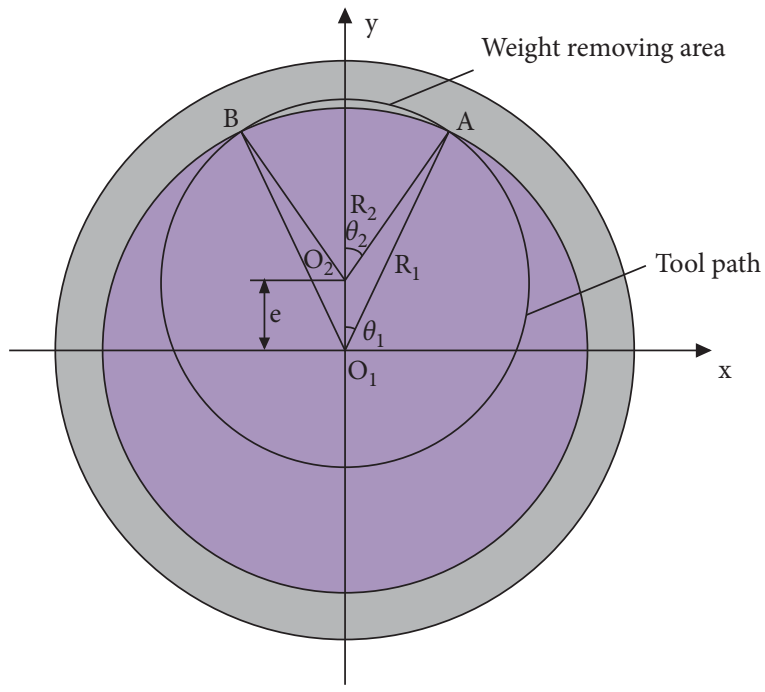

(a)

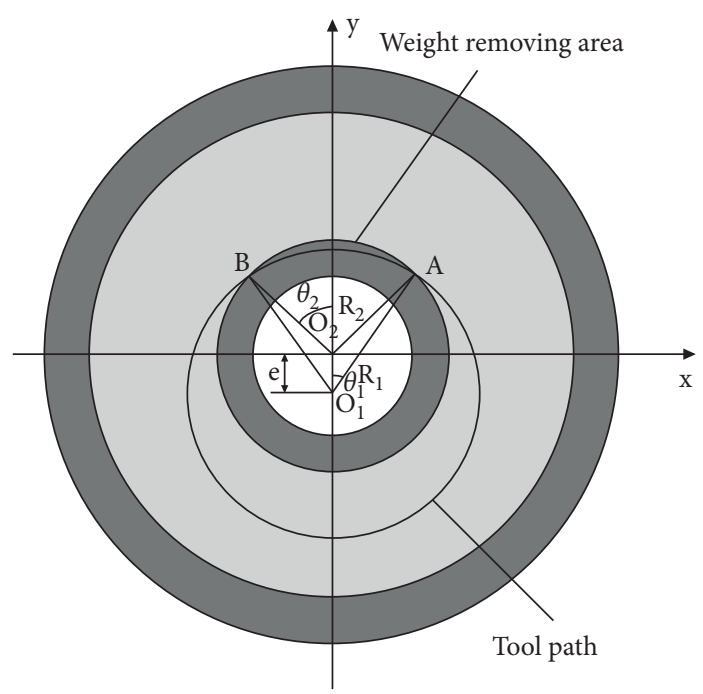

(b)

FIgURe 4: The geometric models of internal and external arc milling: (a) the internal arc milling model; (b) the external arc milling model. 
In Figure 4, the weight removing position is located in the positive direction of $y$-axis, and the principle of axis rotation can be applied to set up the weight removing model when the weight removing position is located in the arbitrary direction.

According to the geometric relations in Figure 4, the weight removing area $(\Delta S)$ can be expressed as follows:

$$
\Delta S=S_{A O_{2} B}-\left(S_{A O_{1} B}-2 S_{\triangle A O_{1} O_{2}}\right) .
$$

$S_{A O_{2} B}=\theta_{2} R_{2}^{2}$ is the area of fan-shaped $\mathrm{AO}_{2} \mathrm{~B}$, and $\theta_{2}=\arccos \left(R_{1}^{2}-e^{2}-R_{2}^{2} / 2 e R_{2}\right) . S_{A O_{1} B}=\theta_{1} R_{1}^{2}$ is the area of fan-shaped $\mathrm{AO}_{1} \mathrm{~B}$, and $\theta_{1}=\arccos \left(e^{2}+R_{1}^{2}-R_{2}^{2} / 2 e R_{1}\right)$.

$S_{\triangle A O_{1} O_{2}}$ and $S_{\triangle B O_{1} O_{2}}$ are the areas of triangles $A_{1} \mathrm{O}_{2}$ and $\mathrm{BO}_{1} \mathrm{O}_{2}$, respectively, and $S_{\triangle A O_{1} O_{2}}=S_{\triangle B O_{1} O_{2}}=$ $\sqrt{p(p-e)\left(p-R_{1}\right)\left(p-R_{2}\right)}, p=\left(e+R_{1}+R_{2}\right) / 2$.
Based on above parameters, (7) can be expressed in

$$
\begin{aligned}
\Delta S= & R_{2}^{2} \cdot \operatorname{arecos}\left(\frac{R_{1}^{2}-e^{2}-R_{2}^{2}}{2 e R_{2}}\right)-R_{1}^{2} \arccos \left(\frac{e^{2}+R_{1}^{2}-R_{2}^{2}}{2 e R_{1}}\right) \\
& +2 \sqrt{p(p-e)\left(p-R_{1}\right)\left(p-R_{2}\right)} .
\end{aligned}
$$

In Figure 4, the unbalance mass of workpiece can be removed only when $e+R_{2}>R_{1}$; if the weight removing depth is assumed to be $h$, the weight removing volume $(\Delta V)$ can be expressed in

$$
\begin{aligned}
\Delta V & =h \cdot \Delta S \\
& =h R_{2}^{2} \cdot \arccos \left(\frac{R_{1}^{2}-e^{2}-R_{2}^{2}}{2 e R_{2}}\right)-h R_{1}^{2} \arccos \left(\frac{e^{2}+R_{1}^{2}-R_{2}^{2}}{2 e R_{1}}\right)+2 h \sqrt{p(p-e)\left(p-R_{1}\right)\left(p-R_{2}\right)} .
\end{aligned}
$$

Equation (9) indicates that the radius of circular tool path can be calculated when the parameters $h$ and $e$ have been set firstly. The value of eccentric distance $(e)$ is bigger, the radius of circular tool path is smaller, and the weight removing area is more concentrative. On the contrary, the weight removing area may be decentralized if the eccentric distance $(e)$ is set to a small value. In practical application, the radius of circular tool path can be set firstly, and then the eccentric distance (e) can be calculated based on (9).

Based on above parameter calculation, the milling spindle with tool is adjusted to the proper position and controlled to remove suitable material, and the unbalance vector of workpiece can be corrected.

\section{The Weight Removing Control Strategies for Unbalance Workpiece}

According to the estimated unbalance vector and the weight removing model, the simple weight removing strategy can be used to remove the equivalent material in workpiece directly. In practical applications, influenced by the measuring and correcting errors, the residual unbalance vector may be greater and still exceed the allowable range after one-time weight removing. Meanwhile, because the weight removing correction belongs to the nonrenewable way, the workpiece characteristics of strength, appearance, assembling, etc. may be damaged when the excessive material is removed in the same position. In consideration of the two problems, an iterative correction method with variable weight removing rate is proposed in the paper.

According to the principle of iterative control method, the true influence coefficient of dynamic balance system is assumed to be $\vec{C}$, the comprehensive error is assumed to be $\vec{e}$, the correcting vector of $k$ th iterative correction is assumed to be $\vec{P}_{k}$, and the unbalance vibration vector of spindle before the $k$ th iterative correction is assumed to be $\vec{V}_{k}$. The following equation can be obtained based on the influence coefficient principle:

$$
\begin{aligned}
\vec{V}_{k} & =\vec{C} \cdot \vec{P}_{k}+\vec{e}, \\
\vec{e} & =\vec{V}_{k}-\vec{C} \cdot \vec{P}_{k} .
\end{aligned}
$$

Suppose the residual unbalance vibration vector equals zero after $k+1$ th iterative correction; the following equation can be obtained.

$$
\begin{aligned}
\vec{V}_{k+1} & =\overrightarrow{\mathrm{C}} \cdot \vec{P}_{k+1}+\vec{e} \approx 0, \\
\vec{C} \cdot \vec{P}_{k+1} & =-\vec{e} .
\end{aligned}
$$

Control equation (12) can be obtained by comparing (10) with (11).

$$
\begin{aligned}
\vec{C} \cdot \vec{P}_{k+1} & =\vec{C} \cdot \vec{P}_{k}-\vec{V}_{k}, \\
\vec{P}_{k+1} & =\vec{P}_{k}-\vec{C}^{-1} \cdot \vec{V}_{k} .
\end{aligned}
$$

In practical application, it is difficult to obtain the true influence coefficient, but the estimated vector of the influence coefficient can be calculated as follows in the process of iterative control:

$$
\vec{C}_{n e w}=\frac{\left(\vec{V}_{k}-\vec{V}_{k-1}\right)}{\left(\vec{P}_{k}-\vec{P}_{k-1}\right)}
$$

The stability of the iterative control equation (12) depends on the measuring and correcting accuracy; unstable 
control or excessive correction may occur if the system errors are excessive. In order to reduce the excessive correction level, the weight removing rate $\alpha \in(0,1]$ is applied in the iterative control equation (12), and the deformed iterative control equation is shown as follows:

$$
\vec{P}_{k+1}=\vec{P}_{k}-\alpha \vec{C}_{n e w}^{-1} \cdot \vec{V}_{k}
$$

In order to prove the astringency of (14), the system error $\vec{e}$ is expressed in

$$
\vec{e}=\vec{C} \cdot \vec{C}_{m} \cdot \vec{P}_{m}
$$

In (15), $\vec{P}_{m}$ is the correcting error, $\vec{C}$ is the true influence coefficient, $\vec{C}_{m}$ is the imaginary accumulative influence coefficient, and the measuring error is included in $\overrightarrow{\mathrm{C}}_{\mathrm{m}} \cdot \vec{P}_{m}$. Equation (16) can be obtained by inserting (15) into (10).

$$
\begin{aligned}
\vec{V}_{k} & =\vec{C} \cdot \vec{P}_{k}+\vec{C} \cdot \vec{C}_{m} \cdot \vec{P}_{m} \\
& =\vec{C} \cdot\left(\vec{P}_{k}+\vec{C}_{m} \cdot \vec{P}_{m}\right) \\
& =\vec{C} \cdot \vec{W}_{k}, \\
\vec{W}_{k} & =\vec{P}_{k}+\vec{C}_{m} \cdot \vec{P}_{m} .
\end{aligned}
$$

In (16), $\vec{W}_{k}$ is defined as the accumulative correcting vector. Suppose the parameters of $\vec{P}_{m}, \vec{C}$, and $\vec{C}_{m}$ are fixed in the process of one-time iterative control. The accumulative correcting vector of $k+1$ th iterative control can be expressed as

$$
\vec{W}_{k+1}=\vec{P}_{k+1}+\vec{C}_{m} \cdot \vec{P}_{m}
$$

Equation (18) can be obtained by inserting (14) and (16) into (17).

$$
\begin{aligned}
\vec{W}_{k+1} & =\vec{P}_{k}+\vec{C}_{m} \cdot \vec{P}_{m}-\alpha \vec{C}_{\text {new }}^{-1} \cdot \vec{V}_{k} \\
& =\vec{W}_{k}-\alpha \vec{C}_{\text {new }}^{-1} \cdot \vec{C} \cdot \vec{W}_{k} \\
& =\vec{W}_{k} \cdot\left(1-\alpha \vec{C}_{\text {new }}^{-1} \cdot \vec{C}\right) \\
& =\vec{W}_{0} \cdot\left(1-\alpha \vec{C}_{\text {new }}^{-1} \cdot \vec{C}\right)^{k+1} .
\end{aligned}
$$

Equation (18) will be convergent when the system meets the condition of

$$
\begin{gathered}
\left|1-\alpha \vec{C}_{\text {new }}^{-1} \cdot \vec{C}\right|<1, \\
0<\alpha<2 \frac{\vec{C}_{\text {new }}}{\vec{C}} .
\end{gathered}
$$

For easy calculation, the vectors of $\vec{C}$ and $\vec{C}_{\text {new }}^{-1}$ are expressed as the exponential form in

$$
\begin{aligned}
C_{\text {new }} & =\left|C_{\text {new }}\right| e^{-j \varphi_{\text {new }}}, \\
C & =|C| e^{-j \varphi_{r}} .
\end{aligned}
$$

Equation (21) can be obtained by inserting (20) into (19).

$$
0<\alpha<2 \frac{\left|C_{\text {new }}\right|}{|C|} e^{j\left(\varphi_{r}-\varphi_{\text {new }}\right)}=2 \frac{\left|C_{\text {new }}\right|}{|C|}\left[\cos \left(\varphi_{r}-\varphi_{\text {new }}\right)+j \sin \left(\varphi_{r}-\varphi_{\text {new }}\right)\right] .
$$

The value range of $\alpha$ can be obtained from (21) and shown in

$$
0<\alpha<2 \frac{\left|C_{\text {new }}\right|}{|C|} \cos \left(\varphi_{r}-\varphi_{\text {new }}\right) .
$$

It can be seen from (22) that the suitable weight removing rate $(\alpha)$ can be found and the control equation (14) will be stable when the phase difference between the true influence coefficient and the estimated influence coefficient does not exceed the range of $\left(-90^{\circ}, 90^{\circ}\right)$.

\section{The Unbalance Vector Online Measuring and Correcting Experiments of Thin Disc Workpiece}

4.1. The Experimental System Design and Error Analysis. In order to simulate the functions of unbalance adjustment and correction, the step-type thin disc workpiece is designed as Figure 5 shows. In Figure 5, A1 is the unbalance adjustment face, and $\mathrm{A} 2$ is the unbalance correction face. In face A1, four bolt-nut groups are distributed uniformly in the radial directions, and the expected unbalance vector in the face can be gained by properly adjusting the corresponding nuts positions of bolt-nut groups. As a similar principle, the expected correcting vector can be gained in face A2.

In Figure 5, the diameter-thickness ratio of workpiece is larger, and the equivalent unbalance vector, composed of the unbalance vectors of face $\mathrm{A} 1$ and $\mathrm{A} 2$, can be taken as distributed in one face. The experimental system is shown in Figure 6(a), and the vector relations of measuring and correcting errors are shown in Figure 6(b) according to the experimental system.

In Figure 6(a), the experimental system is composed of testing-bed, spindle, chuck, supporting system, servo motor system, signal measuring system, testing workpiece, etc. The spindle system can be driven by servo motor system, and the testing speed can be regulated. The testing workpiece can be installed on the end face of chuck and rotated synchronously with spindle. The vibration displacement signals of the front and back ends of spindle can be measured by the eddy current sensors (ML33-02-00-03, made in China), and the reference signal of spindle can be measured by an 


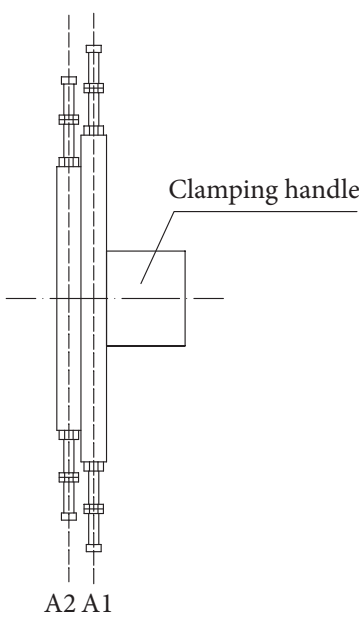

(a)

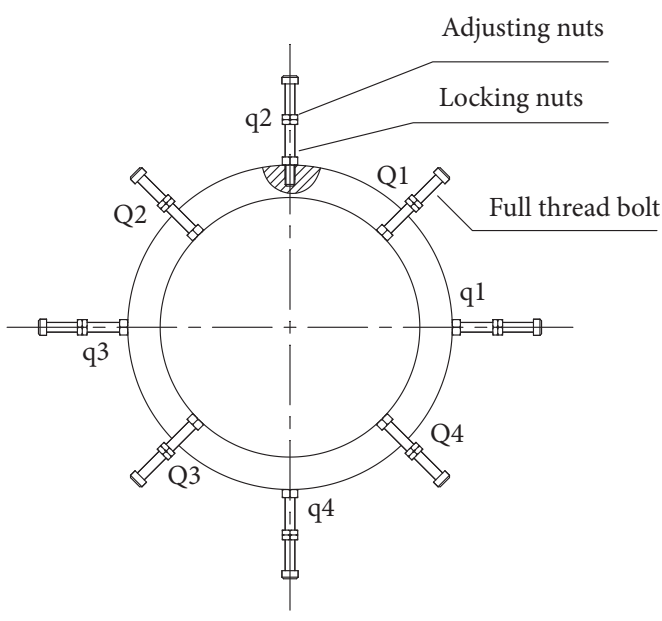

(b)

Figure 5: The structure of experimental workpiece: (a) the main view of workpiece; (b) the left view of workpiece.

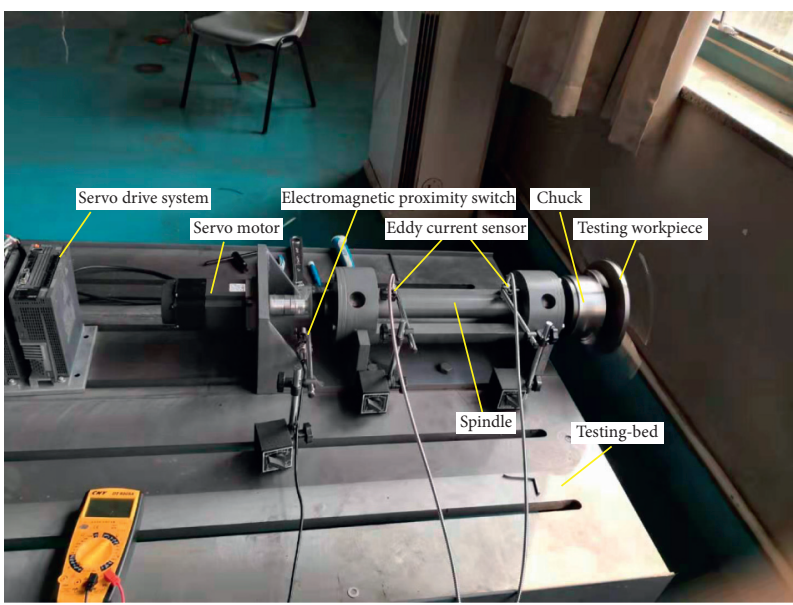

(a)

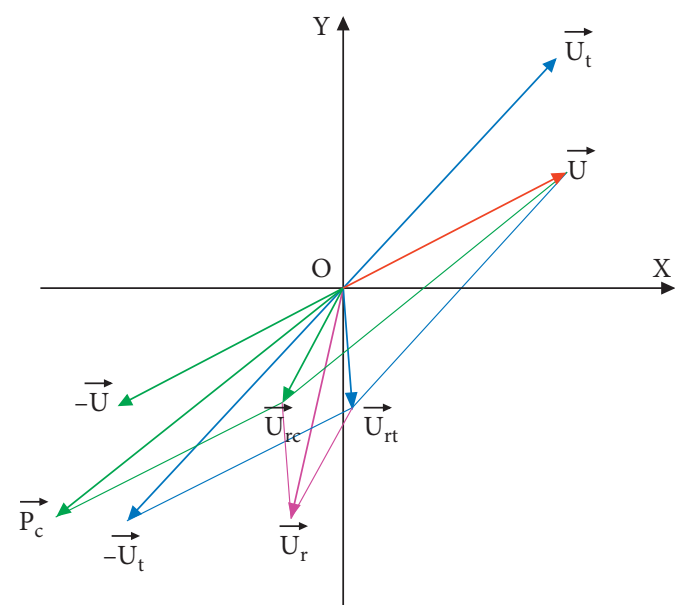

(b)

FIGURE 6: The experimental system and the vector relations of measuring and correcting errors: (a) the experimental system; (b) the measuring and correcting errors analysis.

electromagnetic proximity switch. The signal acquisition card (NI PCI-4472, made in America) is used to acquire the measuring signals.

In order to analyze the experimental result, the measuring and correcting errors are analyzed firstly. In Figure $6(\mathrm{~b}), \vec{U}$ is the true unbalance vector, $\vec{U}_{t}$ is the measuring and calculating value of unbalance vector, and $\vec{P}_{c}$ is the correcting vector.

If the correcting vector is exact $\left(\vec{P}_{c}=-\vec{U}_{t}\right)$, the main affecting factor of the workpiece balance accuracy is the measuring error (defined as $\vec{U}_{r t}=\vec{U}-\vec{U}_{t}$ ). The measuring error is caused by the sensors nonlinear error, vibration signal processing and unbalance vibration signal separating errors, influence coefficient measuring and calculating errors, spindle system dynamic parameters transformation, etc.

If the measuring and calculating value of unbalance vector is exact $\left(\vec{U}_{t}=\vec{U}\right)$, the main affecting factor of the workpiece balance accuracy is the correcting error (defined as $\vec{U}_{r c}=\vec{U}+\vec{P}_{c}$ ). According to the weight removing principle, the correcting error is caused by the modeling error, the inhomogeneities of materials distribution and workpiece structure, the tool error, the locating and controlling errors, etc.

The synthetic error is composed of the measuring and correcting errors and expressed as $\vec{U}_{r}=\vec{U}_{r t}+\vec{U}_{r c}$. The synthetic error can be taken as the residual unbalance vector of the corrected workpiece.

4.2. The Unbalance Vector Measuring and Correcting Experiments for Thin Disc Workpiece. Based on the experimental system of Figure 6(a), the trial weight experiments are repeatedly carried out, and the experimental results are listed in Table 1 [1]. 
TABLE 1: The experiment data before and after trial weight.

\begin{tabular}{lccc}
\hline Testing times & $\begin{array}{c}\text { The initial unbalance vibration } \\
\text { vector }\left(\vec{V}_{0}\right)\left(\mu \mathrm{m}\left\llcorner^{\circ}\right)\right.\end{array}$ & $\begin{array}{c}\text { The unbalance vibration vector after the first } \\
\text { trial weight }\left(\vec{V}_{1}\right)\left(\mu \mathrm{m} \iota^{\circ}\right)\end{array}$ & $\begin{array}{c}\text { The influence coefficients }(\vec{C}) \\
\left(\mu \mathrm{m} /(\mathrm{g} . \mathrm{cm})\left\llcorner^{\circ}\right)\right.\end{array}$ \\
\hline 1 & $11.315 \angle-42.97$ & $17.745 \angle-39.93$ & $0.425 \angle-30.46$ \\
2 & $11.135 \angle-42.32$ & $17.779 \angle-38.14$ & $0.444 \angle-27.08$ \\
3 & $11.112 \angle-42.83$ & $18.008 \angle-38.87$ & $0.46 \angle-28.62$ \\
4 & $11.352 \angle-43.66$ & $18.128 \angle-39.23$ & $0.453 \angle-27.88$ \\
5 & $11.199 \angle-43.31$ & $17.463 \angle-38.73$ & $0.419 \angle-26.25$ \\
\hline
\end{tabular}

It can be seen from Table 1 that, due to the signal measurement and analysis errors, there are some differences among the influence coefficients of different testing times.

In order to discuss the questions of unbalance vector online measurement and estimation, the adjusting nut, located in the contrary direction of reference point, is screwed out $95 \mathrm{~mm}$, and the equivalent unbalance vector is 28.32 g.cm $\angle 180^{\circ}$ in theory. The estimated unbalance vectors of 5-time testing are listed in Table 2.

According to the estimated unbalance vectors in Table 2, the corresponding adjusting nuts in face A2 are adjusted to balance the unbalance vector in face $\mathrm{A} 1$; the residual unbalance vectors and correcting errors are listed in Table 3.

The following conclusions can be gained by analyzing the data of Tables 2 and 3:

(1) The unbalance vector of disc workpiece can be measured online and estimated by measuring and analyzing the unbalance vibration signal transformations of rotating spindle before and after the workpiece is clamped.

(2) The online correcting accuracy can be assessed according to the amplitude of the residual unbalance vector after the unbalance workpiece was corrected. The influence factors of correcting accuracy include the measuring and correcting errors.

\section{The Simulation Analysis of Iterative Weight Removing Method for Unbalance Workpiece}

Based on the above experimental data, $\overrightarrow{U_{0}}$ $=28.32 \mathrm{~g} . \mathrm{cm} \angle 180^{\circ}$ is assumed to be the initial and true unbalance vector of workpiece. According to the experimental data in Table 2, a random value between 1 and 5 is selected as the amplitude of measuring error, a random value between $-10^{\circ}$ and $-40^{\circ}$ is selected as the phase of measuring error, and then the random measuring error vector $\left(\vec{U}_{r t}\right)$ is set up. According to the experimental data in Table 3, a random value between 3 and 10 is selected as the amplitude of correcting error, a random value between $-110^{\circ}$ and $100^{\circ}$ is selected as the phase of correcting error, and then the random correcting error vector $\left(\vec{U}_{r c}\right)$ is set up. Considering the measuring errors, the measuring unbalance vectors $\left(\overrightarrow{U_{k, b}}\right)$ of workpiece before the $k$ th correction is carried out are expressed in

$$
\overrightarrow{U_{k, b}}=\overrightarrow{U_{k-1, a}}+\overrightarrow{U_{r t, k}} .
$$

In (23), $\overrightarrow{U_{k-1, a}}$ is defined as the true unbalance vector of workpiece for the $k$ th correction. Considering the correcting errors, the actual correcting vector is expressed in

$$
\begin{aligned}
\overrightarrow{P_{k}} & =\overrightarrow{U_{k, b}}+\overrightarrow{U_{r c, k}} \\
& =\overrightarrow{U_{k-1, a}}+\overrightarrow{U_{r t, k}}+\overrightarrow{U_{r c, k}} .
\end{aligned}
$$

In (24), $\overrightarrow{P_{k}}$ is defined as the correcting vectors of $k$ th correction. After the $k$ th correction has been carried out, the residual unbalance vector can be expressed in

$$
\overrightarrow{U_{k, a}}=\overrightarrow{U_{r t, k}}+\overrightarrow{U_{r c, k}} .
$$

It can be seen from (25) that the residual unbalance vector can be taken as the excessive correction and caused by the measuring and correcting errors. The residual unbalance vector is assumed to be the true unbalancing vector of workpiece for the $k+1$ th correction.

It can be seen from (23), (24), and (25) that the randomness of the $k$ th balance target and balance result is objective existence due to the randomness of the $k$ th measuring and correcting errors.

In order to analyze the correcting effect of one-time correction with different weight removing rates, the true unbalance vector of workpiece for the $k$ th correction is assumed to be constant, the correcting calculation is carried out on the corresponding 20 groups' random measuring and correcting errors, and the corresponding calculating results (including the measuring unbalance vectors, the correcting vectors, and the residual unbalance vectors) are drawn in the same polar diagram. Figure 7 can be gained by repeating the above calculation process according to different weight removing rates.

In Figure 7, the weight removing rate is set as 1, 0.7, 0.4, and 0.1 ; the corresponding distributed areas of measuring unbalance vectors are shown as $\mathrm{t} 1, \mathrm{t} 2, \mathrm{t} 3$, and $\mathrm{t} 4$; and the corresponding distributed areas of correcting vectors are shown as A1, A2, A3, and A4. After the correction is carried out one time, the corresponding distributed areas of residual unbalance vector are shown as a1, a2, a3, and a4.

It can be seen from Figure 7 that the amplitude of residual unbalance vector is small when the weight removing rate is equal to 1 , but the amplitude of correcting vector is large. For some workpieces, the workpiece performances of strength, appearance, or assembling may be damaged if the excessive weight removing value is focused on one place. The amplitude of correcting vector can be reduced by setting the proper weight removing rate, but the amplitude of residual unbalance vector is large. If the weight removing rate is too 
TABLE 2: The estimated unbalance vectors of 5-time testing.

\begin{tabular}{lccc}
\hline $\begin{array}{l}\text { Testing } \\
\text { times }\end{array}$ & $\begin{array}{c}\text { The unbalance vibration vector after the second } \\
\text { trial weight }(\vec{V})\left(\mu \mathrm{m} \angle^{\circ}\right)\end{array}$ & $\begin{array}{c}\text { The estimated unbalance vectors and measuring errors } \\
\text { The estimated unbalance vectors } \\
\left(\vec{U}_{t}\right)\left(\mathrm{g} \cdot \mathrm{cm} \angle^{\circ}\right)\end{array}$ & $\begin{array}{c}\text { The measuring and calculating errors } \\
\left(\vec{U}_{r t}\right)\left(\mathrm{g} . \mathrm{cm} \angle^{\circ}\right)\end{array}$ \\
\hline 1 & $2.707 \angle-174.41$ & $31.229 \angle 176.29$ & $3.488<-35.38$ \\
2 & $2.800 \angle-153.38$ & $29.594 \angle 179.54$ & $1.295 \angle-10.53$ \\
3 & $2.770 \angle-158.92$ & $30.074 \angle 178.74$ & $1.868<-20.8$ \\
4 & $2.707 \angle-169.52$ & $30.866 \angle 177.04$ & $2.969 \angle-32.47$ \\
5 & $2.927 \angle-180.39$ & $32.059 \angle 175.85$ & $4.328 \angle-32.38$ \\
\hline
\end{tabular}

TABLE 3: The online correcting results for unbalance workpiece.

\begin{tabular}{lcccc}
\hline $\begin{array}{l}\text { Testing } \\
\text { times }\end{array}$ & $\begin{array}{c}\text { The residual unbalance } \\
\text { vibration vectors }\left(\mu \mathrm{m} \angle^{\circ}\right)\end{array}$ & $\begin{array}{c}\text { The equivalent residual unbalance } \\
\text { vectors }\left(\vec{U}_{r}\right)\left(\mathrm{g} \cdot \mathrm{cm} \angle^{\circ}\right)\end{array}$ & $\begin{array}{c}\text { The correcting errors } \\
\left(\vec{U}_{r c}\right)\left(\mathrm{g} . \mathrm{cm} \angle^{\circ}\right)\end{array}$ & $\begin{array}{c}\text { The equivalent correcting } \\
\text { vectors }\left(\vec{P}_{c}\right) \\
\left(\mathrm{g} . \mathrm{cm} \angle^{\circ}\right)\end{array}$ \\
\hline 1 & $13.305 \angle-57.71$ & $8.766 \angle-77.87$ & $6.627 \angle-98.7$ & $28.092 \angle-13.48$ \\
2 & $12.485 \angle-51.46$ & $4.977 \angle-73.25$ & $4.532 \angle-87.97$ & $28.838<-9.04$ \\
3 & $11.712 \angle-28.94$ & $6.685 \angle 76.53$ & $7.166<91.51$ & $29.029 \angle 14.29$ \\
4 & $11.927 \angle-27.56$ & $7.476 \angle 74.19$ & $8.799 \angle 93.05$ & $29.205 \angle 17.51$ \\
5 & $13.035 \angle-52.73$ & $6.330 \angle-67.78$ & $3.760 \angle-109.6$ & $27.29 \angle-7.46$ \\
\hline
\end{tabular}

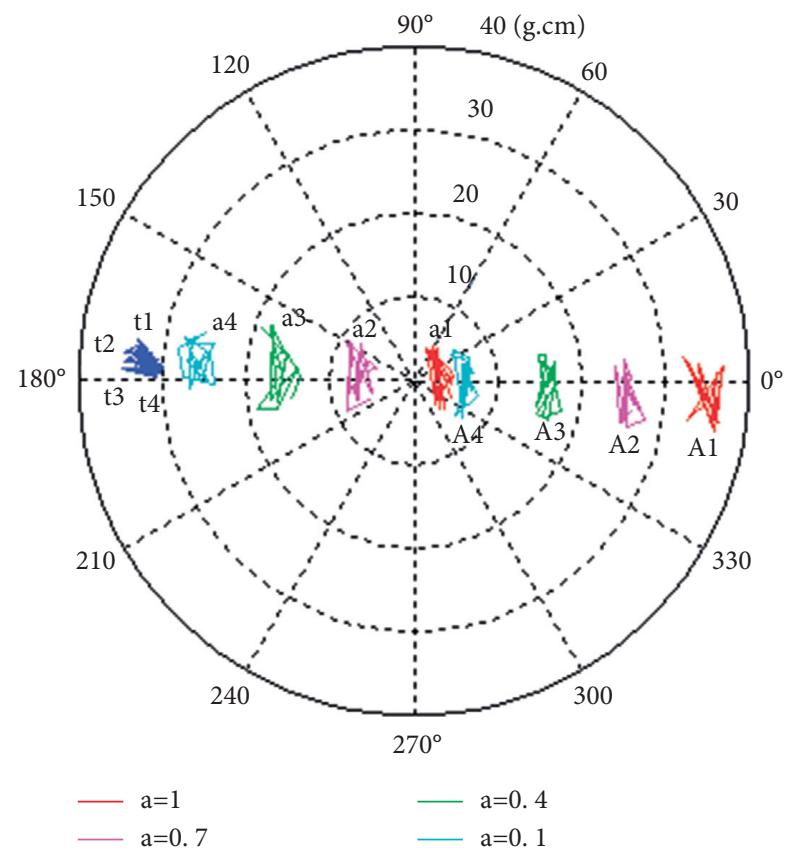

Figure 7: The one-time correcting effect of different weight removing rates.

low, the ratio of the effective removing value and noise is too low, and the correcting effect is unapparent.

The above analysis indicates that the amplitude of correcting vector can be deduced by setting the proper weight removing rate, and the problem of the excessive residual unbalance vector can be resolved by iterative weight removing method. Based on the above method, the residual unbalance vector with maximum amplitude among the 20time repetitive correcting calculations for the $k$ th correction is selected as the balance target for the $k+1$ th iterative calculations. The iterative correcting calculations are carried out until the maximum amplitude of residual unbalance vector does not exceed the permitted value. The distributed areas of correcting vectors and residual unbalance vectors of the iterative corrections with weight removing rates of 0.7 and 0.4 are shown in Figure 8.

In Figure 8(a), the corresponding distribution areas of measuring unbalance vectors, correcting vectors, and residual unbalance vector of the 1st iterative calculations are, respectively, shown as bt1, B1 and b1. Similarly, the parameter distribution areas of the 2nd iterative calculations are, respectively, shown as bt2, B2, and b2; the parameter distribution areas of the 3 rd iterative calculations are, respectively, shown as bt3, B3, and b3. 


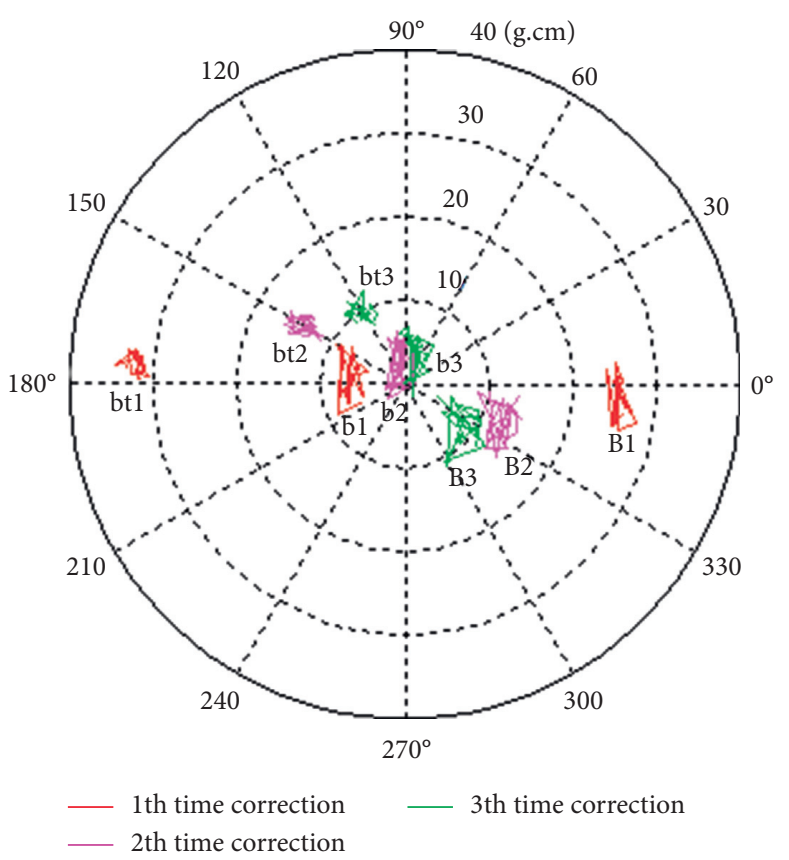

(a)

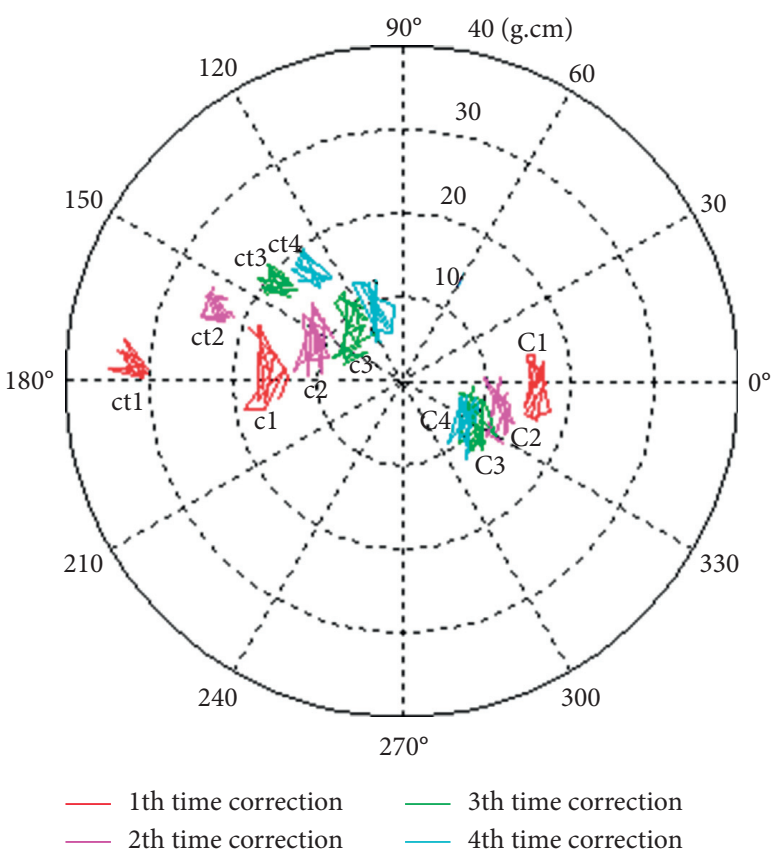

(b)

FIGURE 8: The iterative correcting effect with fixed weight removing rate: (a) the weight removing rate is set as 0.7 ; (b) the weight removing rate is set as 0.4 .

In Figure 8(b), the similar parameter distribution areas of the 1st iterative calculations are, respectively, shown as ct1, C1, and c1; the parameter distribution areas of the 2 nd iterative calculations are, respectively, shown as ct2, C2, and c2; the parameter distribution areas of the 3rd iterative calculations are, respectively, shown as ct3, C3, and c3.

Comparing Figures $8(\mathrm{a})$ and $8(\mathrm{~b})$ with Figure 7 , the following conclusions can be gained:

The value of weight removing rate is larger, the maximum balance accuracy is higher, and the iterative times are less. If the weight removing rate is 1 , the maximum balance accuracy can be realized by one-time iterative correction. If the weight removing rate is 0.7 , the maximum balance accuracy can be realized by two-time iterative correction. If the weight removing rate is 0.4 , the maximum balance accuracy can be realized by three-time iterative correction.

In order to reduce the correcting vector amplitude of firsttime correction and obtain the highest correcting accuracy at the same time, the iterative correction method with variable weight removing rate is proposed in the paper. The weight removing rate of the first-time correction can be set as low so as to reduce the correcting vector amplitude of first-time correction, and the weight removing rate of the last-time correction is set as 1 so as to improve the correcting accuracy. Based on the proposed method, the highest correcting accuracy can be obtained by two- or three-time iterative correction. For example, the weight removing rates of the three-time iterative correction are, respectively, set as $0.4,0.7$, and 1 ; the corresponding distributed areas of correcting vectors and residual unbalance vectors are shown in Figure 9.

In Figure 9, the parameter distribution areas of measuring unbalance vectors, correcting vectors, and residual

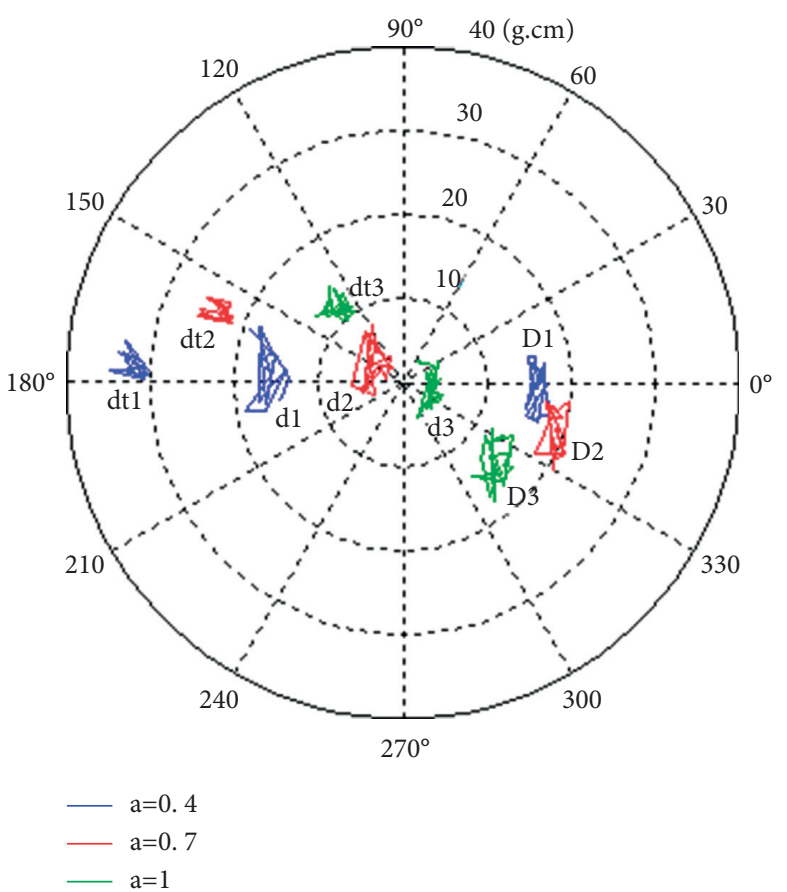

FIgURE 9: The iterative correcting effect with variable weight removing rates.

unbalance vector of the 1st iterative calculations are, respectively, shown as dt1, D1, and d1. Similarly, the parameter distribution areas of the 2 nd iterative calculations are, respectively, shown as $\mathrm{dt} 2, \mathrm{D} 2$, and $\mathrm{d} 2$; the parameter distribution areas of the $3 \mathrm{rd}$ iterative calculations are, respectively, shown as dt3, D3, and d3. 
The following conclusions can be gained by analyzing Figure 9:

(1) The vector amplitude of first-time correction has been deduced greatly, and the ideal correcting accuracy has been obtained by three-time iterative correction

(2) To ensure that the correcting vector amplitude of the three-time iterative correction is average, the weight removing rates should be properly set

(3) By comparing the positions of $\mathrm{dt} 1, \mathrm{dt} 2$, and $\mathrm{dt} 3$, it can be found that the larger amplitude of the residual unbalance vector is usually accompanied by large phase deviation, the correcting directions of the three-time iteration can be separated, and the phenomenon of repeated weight removing in the same direction can be avoided

\section{Conclusions}

Aiming at solving the unbalance correction problems of thin disc workpiece, this paper discusses the weight removing model and the online weight removing strategies. The following conclusions are confirmed by theory, experiments, and simulation analysis:

(1) In practical application, the measuring and correcting errors are unavoidable, the excessive correction may be produced based on the traditional weight removing method, and the workpiece may be damaged and non-revived

(2) The advantages of the proposed iterative correction method with variable weight removing rate have been proved by simulation and calculation methods, the correcting vector amplitude of first-time correction can be reduced, and the highest correcting accuracy can be obtained at the same time

\section{Data Availability}

Some experimental data have been published in [11].

\section{Conflicts of Interest}

The authors declare no conflicts of interest with respect to the research, authorship, and/or publication of this article.

\section{Acknowledgments}

This work was supported by the Science and Technology Funds from Tianjin Municipal Education Commission (No. 2017KJ110).

\section{References}

[1] S. H. Zhang and Z. M. Zhang, "Online measuring and estimating methods for the unbalancing vector of thin-disc workpiece based on the adaptive influence coefficient," Journal of Vibration and Control, vol. 27, no. 15-16, pp. 1753-1764, 2021.
[2] M. Stoesslein, D. A. Axinte, and A. Bilbao Guillerna, "Pulsed laser ablation as a tool for in-situ balancing of rotating parts," Mechatronics, vol. 38, pp. 54-67, 2016.

[3] M. Stoesslein, D. A. Axinte, and D. Gilbert, "On-the-fly laser machining: a case study for in situ balancing of rotative parts," Journal of Manufacturing Science and Engineering, vol. 139, no. 3, pp. 1-14, 2017.

[4] Z. Zeng, Z. Liu, and Y. Ren, "Modeling study of V-shaped milling unbalance correction of automatic balancing machine for motor armature," Journal of Mechanical Engineering, vol. 46, no. 7, pp. 187-192, 2010.

[5] S. M. Yin, Y. Jiang, W. J. Lu, J. P. Zhu, Z. S. Wang, and J. D. Meng, "Modeling study of R-shaped milling unbalance correction of balancing machine for rotor," Modular Machine Tool \& Automatic Manufacturing Technology, vol. 9, pp. 39-41, 2014.

[6] Y. A. Khulief, W. Oke, and M. A. Mohiuddin, "Modally tuned influence coefficients for low-speed balancing of flexible rotors," Journal of Vibration and Acoustics-Transactions of the ASME, vol. 136, no. 2, pp. 858-862, 2013.

[7] J. Zhang, J. W. Wu, and Z. Y. Ma, "Hierarchical bayesian calibration and on-line updating method for influence coefficient of automatic dynamic balancing machine," Chinese Journal of Mechanical Engineering, vol. 22, no. 6, pp. 876-882, 2009.

[8] H. W. Fan, B. J. Shi, and M. Q. Jing, "Modeling and simulation of influence coefficient table method for rotor single-plane online active balancing," Mechanical Science and Technology for Aerospace Engineering, vol. 37, no. 8, pp. 1195-1199, 2018.

[9] L. Zhang, L. Luo, and J. Xu, "An improved adaptive control method for active balancing control of rotor with time-delay," IEICE Electronics Express, vol. 14, no. 23, 2017.

[10] F. B. Becker, M. A. Sehr, and S. Rinderknecht, "Vibration isolation for parameter-varying rotor systems using piezoelectric actuators and gain-scheduled control," Journal of Intelligent Material Systems and Structures, vol. 28, no. 16, pp. 2286-2297, 2017.

[11] J. Xu, Y. Zhao, Z. Y. Jia, and J. J. Zhang, "Rotor dynamic balancing control method based on fuzzy auto-tuning single neuron PID," IEICE Electronics Express, vol. 14, no. 10, 2017. 